MMC-M-11

November 1997

\title{
Third Quantization of Kaluza-Klein Cosmology and Compactification
}

\author{
Yoshiaki OHKUWA 用 \\ Department of Mathematics, Miyazaki Medical College, Kiyotake, \\ Miyazaki 889-16, Japan
}

\begin{abstract}
We study on the third quantization of a Kaluza-Klein toy model. In this model time $(x)$ is defined by the scale factor of universe, and the space coordinate $(y)$ is defined by the ratio of the scales of the ordinary space and the internal space. We calculate the number density of the universes created from nothing and examine whether the compactification can be explained statistically by the idea of the third quantization.
\end{abstract}

*E-mail address: ohkuwa@macqm.miyazaki-med.ac.jp 


\section{Introduction}

The problem of time is now considered as one of the deepest problems in quantum cosmology. ${ }^{[1]}$ It has many complicated aspects and is still controversial, though many ideas have been proposed to solve it. ${ }^{[1,2]}$ Usually, the Wheeler-DeWitt equation is considered as the fundamental equation in quantum cosmology. ${ }^{[3]}$ However, because the Wheeler-DeWitt equation is a hyperbolic second-order differential equation (the Klein-Gordon type), there is a problem in the naive interpretation that $|\Psi|^{2}$ is a probability, where $\Psi$ is a solution to the Wheeler-DeWitt equation. One of the proposed ideas to solve this problem is the third quantization in analogy with the second quantization of the Klein-Gordon equation $\cdot^{[4-15]}$

The Kaluza-Klein theory is one of unified theories of gravity and matter fields. ${ }^{[16]}$ In this theory it is assumed that the space-time has higher dimensions, the higher-dimensional space is a product of ordinary (external) space and internal space, and the latter is small, which is called compactification. The gravitational field and matter field are contained in the metric tensors of the higherdimensional space-time. The quantum cosmology of the Kaluza-Klein theory has been studied by many authors, ${ }^{[17-22]}$ and the third quantization of it has also been studied.$^{[23-26]}$ However, as far as the present author knows, the idea of the third quantization has not been utilized directly to explain the compactification.

In this paper we will examine the third quantization of a Kaluza-Klein cosmology, in which time $(x)$ is defined by the scale factor of universe, and the space coordinate $(y)$ is defined by the ratio of the scale of the ordinary space and that of the internal space. And we will calculate the number density of the universes created from nothing. The compactification could be explained statistically, if many of universes created from nothing had such $y$ that means compactification. We will find that there is a possibility to explain the compactification, when both the external and internal spaces are three-dimensional flat space $R^{3}$.

In $\S 2$ we will consider the quantum cosmology of a Kaluza-Klein toy model,

which will be third quantized in $\S 3$. In $\S 4$ we will calculate the number density of universes created from nothing, and in $§ 5$ we will examine the possibility to ex- 
plain the compactification statistically through the idea of the third quantization. We summarize in $\S 6$.

\section{Quantum Cosmology of Kaluza-Klein Toy Model}

Let us start from a $(1+\mathrm{n}+\mathrm{m})$-dimensional space-time. We consider the following minisuperspace model in which the $(\mathrm{n}+\mathrm{m})$-dimensional space is a product of a space with $\mathrm{n}$ dimensions and a space with $\mathrm{m}$ dimensions. ${ }^{[17,18,23-26]}$ The metric is assumed to be

$$
\begin{aligned}
d s^{2} & =g_{M N} d x^{M} d x^{N} \\
& =-N^{2}(t) d t^{2}+a^{2}(t) \tilde{g}_{\mu \nu} d x^{\mu} d x^{\nu}+b^{2}(t) \hat{g}_{m n} d x^{m} d x^{n} .
\end{aligned}
$$

Here $N(t)$ is the lapse function, $a(t)$ and $b(t)$ are the scale factors of the two spaces, $g_{M N}$ are the $(1+\mathrm{n}+\mathrm{m})$-dimensional metric tensors, and $\tilde{g}_{\mu \nu}, \hat{g}_{m n}$ are metric tensors of $M^{n}, M^{m}$, respectively, where $M^{n}$ is $S^{n}, R^{n}$ or $H^{n}$. The Einstein action with a cosmological constant $\Lambda$ is written as

$$
S=\int d^{1+n+m} x \mathcal{L}, \quad \mathcal{L}=\frac{1}{16 \pi G} \sqrt{-g}(R-2 \Lambda)
$$

Substituting Eqs. (1) into Eqs. (2), we have

$$
\begin{aligned}
S=\int d t L, & \\
L=\frac{v_{n m}}{16 \pi G} N a^{n} b^{m} & {\left[n(n-1)\left(\frac{k_{n}}{a^{2}}-\frac{\dot{a}^{2}}{N^{2} a^{2}}\right)+m(m-1)\left(\frac{k_{m}}{b^{2}}-\frac{\dot{b}^{2}}{N^{2} b^{2}}\right)\right.} \\
& \left.\quad-\frac{2 n m \dot{a} \dot{b}}{N^{2} a b}-2 \Lambda\right]
\end{aligned}
$$

where $v_{n m}=\int d^{n+m} x \sqrt{\tilde{g} \hat{g}}, \quad \tilde{g}=\operatorname{det} \tilde{g}_{\mu \nu}, \quad \hat{g}=\operatorname{det} \hat{g}_{m n}, \quad \dot{a}=\frac{d a}{d t}$ and $k_{n}=1,0,-1$ when $M^{n}$ is $S^{n}, R^{n}, H^{n}$, respectively.

Since the action (3) is not diagonal with respect to $a, b$, we change variables as

$$
a=r \gamma^{m}, \quad b=r \gamma^{-n}
$$


where $r$ is a scale factor and $\gamma$ determines the ratio of $a / b$. With these variables the Lagrangian (3) becomes

$$
\begin{aligned}
L & =-\frac{c_{r}}{2} \frac{r^{n+m-2}}{N} \dot{r}^{2}+\frac{c_{\gamma}}{2} \frac{r^{n+m}}{N} \frac{\dot{\gamma}^{2}}{\gamma^{2}}-N U, \\
c_{r} & =\frac{2 v_{n m}}{16 \pi G}(n+m)(n+m-1), \quad c_{\gamma}=\frac{2 v_{n m}}{16 \pi G} n m(n+m), \\
U & =-\frac{v_{n m}}{16 \pi G}\left\{r^{n+m-2}\left[k_{n} n(n-1) \gamma^{-2 m}+k_{m} m(m-1) \gamma^{2 n}\right]-2 \Lambda r^{n+m}\right\} .
\end{aligned}
$$

Then the Hamiltonian reads

$$
\begin{aligned}
H & =N \mathcal{H}, \\
\mathcal{H} & =-\frac{1}{2 c_{r}} \frac{p_{r}^{2}}{r^{n+m-2}}+\frac{\gamma^{2}}{2 c_{\gamma}} \frac{p_{\gamma}^{2}}{r^{n+m}}+U,
\end{aligned}
$$

where $p_{r}=\frac{\partial L}{\partial \dot{r}}=-c_{r} N^{-1} r^{n+m-2} \dot{r}, \quad p_{\gamma}=\frac{\partial L}{\partial \dot{\gamma}}=c_{\gamma} N^{-1} r^{n+m} \gamma^{-2} \dot{\gamma}$.

From the Hamiltonian constraint $\mathcal{H} \approx 0$, we obtain the Wheeler-DeWitt equation,

$$
\begin{gathered}
{\left[r^{-P} \frac{\partial}{\partial r} r^{P} \frac{\partial}{\partial r}-\frac{c_{r}}{c_{\gamma}} \frac{\gamma^{2-Q}}{r^{2}} \frac{\partial}{\partial \gamma} \gamma^{Q} \frac{\partial}{\partial \gamma}+V\right] \Psi(r, \gamma)=0} \\
V=-\frac{2 c_{r} v_{n m}}{16 \pi G}\left\{r^{2(n+m-2)}\left[k_{n} n(n-1) \gamma^{-2 m}+k_{m} m(m-1) \gamma^{2 n}\right]-2 \Lambda r^{2(n+m-1)}\right\}
\end{gathered}
$$

where $\Psi(r, \gamma)$ is a wave function of universe, $P, Q$ are parameters of operator ordering. Changing variables by

$$
r=e^{x}, \quad \gamma=e^{y},
$$

where $x$ and $y$ determine the scale of universe and the ratio of two spaces, respectively, we obtain

$$
\left\{\frac{\partial^{2}}{\partial x^{2}}+(P-1) \frac{\partial}{\partial x}-\frac{c_{r}}{c_{\gamma}}\left[\frac{\partial^{2}}{\partial y^{2}}+(Q-1) \frac{\partial}{\partial y}\right]+e^{2 x} V\right\} \Psi(x, y)=0 .
$$

If we choose $P=1, Q=1$, Eq. (8) becomes

$$
\left[\frac{\partial^{2}}{\partial x^{2}}-\frac{c_{r}}{c_{\gamma}} \frac{\partial^{2}}{\partial y^{2}}+e^{2 x} V\right] \Psi(x, y)=0 .
$$


The Wheeler-DeWitt equation (9) is the Klein-Gordon type, and $|\Psi|^{2}$ is not conserved. Therefore, there is a difficulty in the naive interpretation that $|\Psi|^{2}$ is a probability. We will investigate the third quantization of this model in the next section.

\section{Third Quantization}

Let us regard $x$ as time and $y$ as the space coordinate. The third quantized action to yield the Wheeler-DeWitt equation (9) is

$$
\begin{aligned}
S_{3 Q} & =\int d x d y \mathcal{L}_{3 Q}, \\
\mathcal{L}_{3 Q} & =\frac{1}{2}\left[\left(\frac{\partial \Psi}{\partial x}\right)^{2}-\frac{c_{r}}{c_{\gamma}}\left(\frac{\partial \Psi}{\partial y}\right)^{2}-e^{2 x} V \Psi^{2}\right] .
\end{aligned}
$$

The canonical momentum is given by

$$
\Pi_{\Psi}=\frac{\partial \mathcal{L}_{3 Q}}{\partial \frac{\partial \Psi}{\partial x}}=\frac{\partial \Psi}{\partial x},
$$

and the Hamiltonian reads

$$
\mathcal{H}_{3 Q}=\frac{1}{2}\left[\Pi_{\Psi}^{2}+\frac{c_{r}}{c_{\gamma}}\left(\frac{\partial \Psi}{\partial y}\right)^{2}+e^{2 x} V \Psi^{2}\right] .
$$

To quantize this model, we impose the canonical commutation relations

$$
\begin{aligned}
{\left[\Psi(x, y), \Pi_{\Psi}\left(x, y^{\prime}\right)\right] } & =i \delta\left(y-y^{\prime}\right) \\
{\left[\Psi(x, y), \Psi\left(x, y^{\prime}\right)\right] } & =\left[\Pi_{\Psi}(x, y), \Pi_{\Psi}\left(x, y^{\prime}\right)\right]=0 .
\end{aligned}
$$

Let us write a complete set of normalized positive frequency solutions of Eq. (9) as $\left\{u_{p}(x, y)\right\}$, where $p$ labels the mode function and $u_{p}$ satisfies the normalization condition,

$$
i \int d y\left(u_{p}^{*} \frac{\partial}{\partial x} u_{q}-u_{q} \frac{\partial}{\partial x} u_{p}^{*}\right)=\delta(p-q) .
$$

Using these normal modes, we expand $\Psi(x, y)$ as

$$
\Psi(x, y)=\int d p\left[a_{p} u_{p}(x, y)+a_{p}^{\dagger} u_{p}^{*}(x, y)\right]
$$


where $a_{p}$ and $a_{p}^{\dagger}$ satisfy

$$
\left[a_{p}, a_{q}^{\dagger}\right]=\delta(p-q),\left[a_{p}, a_{q}\right]=\left[a_{p}^{\dagger}, a_{q}^{\dagger}\right]=0
$$

Therefore, $a_{p}$ and $a_{p}^{\dagger}$ are annihilation and creation operators of a universe with $p$, respectively. The vacuum state $|0\rangle$ is defined by

$$
a_{p}|0\rangle=0 \quad \text { for } \forall p
$$

and the Fock space is spanned by $a_{p_{1}}^{\dagger} a_{p_{2}}^{\dagger} \cdots|0\rangle$.

\section{Universe Creation from Nothing}

Since the potential $V$ in Eqs. (6) is time $(x)$ and space $(y)$ dependent, universes are created from nothing. ${ }^{[7,10,27]}$ In order to see this and for simplicity, let us consider the case that both the ordinary space and the internal space are flat $\left(k_{n}=0, k_{m}=0\right) \cdot .^{[24-26]}$ We assume that $v_{n m}$ is some properly fixed finite constant. In this case Eq. (9) is

$$
\left[\frac{\partial^{2}}{\partial x^{2}}-\frac{c_{r}}{c_{\gamma}} \frac{\partial^{2}}{\partial y^{2}}+c_{\Lambda} e^{2(n+m) x}\right] \Psi(x, y)=0
$$

with $c_{\Lambda}=\frac{c_{r} v_{n m}}{4 \pi G} \Lambda$. The normal mode function $u_{p}(x, y)$ of Eq. (16) can be calculated as

$$
u_{p}(x, y)=\mathcal{N}_{p} Z_{ \pm \nu}(z) e^{i p y}
$$

where we have assumed $\Lambda>0, z=\frac{\sqrt{c_{\Lambda}}}{n+m} e^{(n+m) x}, \nu=\frac{-i}{n+m} \sqrt{\frac{c_{r}}{c_{\gamma}}}|p|, \mathcal{N}_{p}$ is a normalization factor that satisfies Eq. (12), $Z_{\nu}$ is a Bessel function, and $p$ can be regarded as a canonical momentum of $y$.

We define in-mode function $u_{p}^{i n}(x, y)$ as

$$
\begin{aligned}
u_{p}^{i n}(x, y) & =\mathcal{N}_{p}^{i n} J_{\nu}(z) e^{i p y} \\
\mathcal{N}_{p}^{i n} & =\frac{1}{2 \sqrt{n+m}}\left(\sinh \frac{\pi}{n+m} \sqrt{\frac{c_{r}}{c_{\gamma}}}|p|\right)^{-\frac{1}{2}}
\end{aligned}
$$

which satisfies

$$
u_{p}^{i n}(x, y) \propto \exp \left[-i\left(\sqrt{\frac{c_{r}}{c_{\gamma}}}|p| x-p y\right)\right]
$$


when $x \rightarrow-\infty$. The expansion of $\Psi$ is

$$
\Psi(x, y)=\int d p\left[a_{p}^{i n} u_{p}^{i n}(x, y)+a_{p}^{i n \dagger} u_{p}^{i n^{*}}(x, y)\right]
$$

and the in vacuum $|0, i n\rangle$, which we regard as "nothing", is defined by

$$
a_{p}^{i n}|0, i n\rangle=0 \quad \text { for } \forall p
$$

In the same way we define out-mode function $u_{p}^{\text {out }}(x, y)$ as

$$
\begin{aligned}
u_{p}^{\text {out }}(x, y) & =\mathcal{N}_{p}^{\text {out }} H_{-\nu}^{(2)}(z) e^{\text {ipy }} \\
\mathcal{N}_{p}^{\text {out }} & =\frac{1}{2 \sqrt{2(n+m)}} \exp \left(\frac{\pi}{2(n+m)} \sqrt{\frac{c_{r}}{c_{\gamma}}}|p|\right)
\end{aligned}
$$

which satisfies

$$
u_{p}^{\text {out }}(x, y) \propto \exp \left[-\frac{n+m}{2} x-i\left(\frac{\sqrt{c_{\Lambda}}}{n+m} e^{(n+m) x}-p y\right)\right],
$$

when $x \rightarrow \infty$. The expansion of $\Psi$ is

$$
\Psi(x, y)=\int d p\left[a_{p}^{\text {out }} u_{p}^{\text {out }}(x, y)+a_{p}^{\text {out }}{ }^{\dagger} u_{p}^{\text {out* }}(x, y)\right]
$$

and the out vacuum $\mid 0$, out $\rangle$ is defined by

$$
\left.a_{p}^{\text {out }} \mid 0, \text { out }\right\rangle=0 \quad \text { for } \forall p
$$

The Bogoliubov coefficients $c_{i}(p, q)(i=1,2)$ are defined by

$$
u_{p}^{\text {out }}(x, y)=\int d q\left[c_{1}(p, q) u_{q}^{i n}(x, y)+c_{2}(p, q) u_{q}^{i n^{*}}(x, y)\right]
$$

Using the relation $H_{-\nu}^{(2)}(z)=\frac{i}{\sin \pi \nu}\left[e^{-i \pi \nu} J_{-\nu}(z)-J_{\nu}(z)\right]$ and Eqs. (18), (20), (22), we can calculate $c_{i}(p, q)$ as

$$
\begin{aligned}
c_{1}(p, q) & =\frac{1}{\sqrt{1-\exp \left[-\frac{2 \pi}{n+m} \sqrt{\frac{c_{r}}{c_{\gamma}}}|p|\right]}} \delta(p-q), \\
c_{2}(p, q) & =\frac{-1}{\sqrt{\exp \left[\frac{2 \pi}{n+m} \sqrt{\frac{c_{r}}{c_{\gamma}}}|p|\right]-1}} \delta(p+q) .
\end{aligned}
$$

${ }^{\dagger}$ If we choose $H_{\nu}^{(2)}(z)$ instead of $H_{-\nu}^{(2)}(z)$ in Eqs. (20), Eqs. (21)-(33) will not change, but the adiabatic vacuum $\mid 0$, adia $\rangle$ in Eqs. (36) corresponds to $H_{-\nu}^{(2)}(z)$. 
The number density of the universe with $p$ created from nothing is defined as

$$
N_{p}^{(0)}=\left\langle 0, \text { in }\left|a_{p}^{\text {out }}{ }^{\dagger} a_{p}^{\text {out }}\right| 0, \text { in }\right\rangle
$$

From Eqs. (19), (23), (24) we obtain

$$
N_{p}^{(0)}=\int d q\left|c_{2}(p, q)\right|^{2} \propto \frac{1}{\exp \left[\frac{2 \pi}{n+m} \sqrt{\frac{c_{r}}{c_{\gamma}}}|p|\right]-1},
$$

where we have omitted an irrelevant constant. ${ }^{[24-26]}$ Note that this is a Planck distribution with respect to $|p|$.

\section{Compactification of Internal Space}

Now let us examine whether the compactification can be explained directly from the third quantization or not. In order to know the number density of created universe with respect to $y$, we define the operators $a_{y}^{\text {out }}$ as

$$
a_{y}^{\text {out }}=\frac{1}{\sqrt{2 \pi}} \int d p e^{-i p y} a_{p}^{\text {out }}
$$

which satisfy

$$
\left[a_{y}^{\text {out }}, a_{y^{\prime}}^{\text {out }}{ }^{\dagger}\right]=\delta\left(y-y^{\prime}\right), \quad\left[a_{y}^{\text {out }}, a_{y^{\prime}}^{\text {out }}\right]=\left[a_{y}^{\text {out }}{ }^{\dagger}, a_{y^{\prime}}^{\text {out }}{ }^{\dagger}\right]=0
$$

We can regard $a_{y}^{\text {out }}$ and $a_{y}^{\text {out }}{ }^{\dagger}$ as the annihilation and creation operators of a universe with $y$, respectively. Then the number density with respect to $y$ can be defined by

$$
N_{y}^{(0)}=\left\langle 0, \text { in }\left|a_{y}^{\text {out }} a_{y}^{\text {out }}\right| 0, \text { in }\right\rangle .
$$

Using Eqs. (19),(23),(26),(28), we find

$$
\begin{aligned}
N_{y}^{(0)} & =\frac{1}{2 \pi} \int d p^{\prime} d p e^{i\left(p^{\prime}-p\right) y}\left\langle 0, \text { in }\left|a_{p^{\prime}}^{\text {out }} a_{p}^{\text {out }}\right| 0, \text { in }\right\rangle \\
& =\frac{1}{2 \pi} \int d p^{\prime} d p d q e^{i\left(p^{\prime}-p\right) y} c_{2}\left(p^{\prime}, q\right) c_{2}^{*}(p, q) \\
& =c_{N}
\end{aligned}
$$


where $c_{N}$ is a constant which does not depend on $y$. If we define

$$
a=e^{\alpha}, \quad b=e^{\beta}, \quad \delta=|\alpha-\beta|, \quad \Gamma=e^{\delta}
$$

these equations and Eqs. (4), (7) mean

$$
\alpha-\beta=(n+m) y, \quad \Gamma= \begin{cases}\frac{a}{b} & (a \geq b) \\ \frac{b}{a} & (a \leq b)\end{cases}
$$

that is $\Gamma$ represents the ratio of the scales of the larger space and the smaller space. Then we can calculate the number densities $N_{\delta}^{(0)}, N_{\Gamma}^{(0)}$ with respect to $\delta, \Gamma$ as

$$
\begin{aligned}
& N_{\delta}^{(0)}=N_{y}^{(0)} \frac{d y}{d \delta}+N_{-y}^{(0)} \frac{d y}{d \delta}=\frac{2 c_{N}}{n+m} \quad(y \geq 0), \\
& N_{\Gamma}^{(0)}=N_{\delta}^{(0)} \frac{d \delta}{d \Gamma}=\frac{2 c_{N}}{n+m} \frac{1}{\Gamma} \quad(\Gamma \geq 1) .
\end{aligned}
$$

Hence, for any finite $\Gamma_{0}$, we obtain

$$
\frac{\operatorname{Prob}\left(\Gamma_{0} \leq \Gamma<\infty\right)}{\operatorname{Prob}\left(1 \leq \Gamma \leq \Gamma_{0}\right)}=\lim _{\Gamma_{1} \rightarrow \infty} \frac{\int_{\Gamma_{0}}^{\Gamma_{1}} d \Gamma \frac{1}{\Gamma}}{\int_{1}^{\Gamma_{0}} d \Gamma \frac{1}{\Gamma}}=\lim _{\Gamma_{1} \rightarrow \infty} \frac{\ln \Gamma_{1}-\ln \Gamma_{0}}{\ln \Gamma_{0}} \rightarrow \infty
$$

where Prob is a probability. This result means that many of the universes created from nothing have a large ratio of the size of two spaces. Note that in this toy model the two spaces are completely symmetric. Therefore, if we assume $n=m$ $=3\left(M^{3}=R^{3}\right)$ and regard the greater space as the ordinary (external) space and the smaller space as the internal space, there seems to be a possibility that the compactification can be explained statistically.f

However, there remain some problems in the above discussion. First, let us take another model where, for example, $\mathrm{n}=3, \mathrm{~m}=1$, the three-dimensional space is flat $\left(M^{3}=R^{3}, k_{3}=0\right)$ and the one-dimensional space is a circle $\left(M^{1}=S^{1}, k_{1}=\right.$ 1). In this case Eq. (9) becomes

$$
\left[\frac{\partial^{2}}{\partial x^{2}}-\frac{\partial^{2}}{\partial y^{2}}+c_{\Lambda} e^{8 x}\right] \Psi(x, y)=0
$$

\footnotetext{
${ }^{\ddagger}$ Many ideas have been proposed to explain the compactification, ${ }^{[16]}$ but this possibility is a new one to explain it statistically. ${ }^{[28]}$
} 
with $c_{\Lambda}=\frac{3}{8}\left(\frac{v_{n m}}{\pi G}\right)^{2} \Lambda$, and the same results as Eqs. (29), (33) hold. In this model we must regard the flat space $R^{3}$ as the external space and the circle $S^{1}$ as the internal space. Then Eqs. (29), (33) mean that there are both many universes which are compactified and those which are not compactified. Therefore, our discussion is model dependent. If we will be also able to obtain the same result as Eqs. (33), in a more realistic model, for example, with $\mathrm{n}=\mathrm{m}=3$ and $M^{3}=S^{3}, k_{3}=1$, then there will be a possibility to explain the compactification statistically in this case. It will be also interesting if the compactification can be explained when the space-time has the topology $R \times S^{3} \times S^{3} \times S^{3}$ in ten dimensions. In this case, it will be required that one space $S^{3}$ is large and two other spaces $S^{3} \times S^{3}$ are small. So it seems that further investigation will be necessary on more realistic models.

Second, thus far we have interpreted Eq. (16) as a field equation in a flat metric, $d s_{3 Q}^{2}=-d x^{2}+d \tilde{y}^{2}, \quad \tilde{y}=\sqrt{\frac{c_{\gamma}}{c_{r}}} y$, with a time dependent potential $c_{\Lambda} e^{2(n+m) x}$. However, Eq. (16) can be also regarded as a field equation with a mass $\sqrt{c_{\Lambda}}$ in the Milne metric,

$$
\begin{aligned}
d s_{3 Q}^{2} & =e^{2(n+m) x}\left(-d x^{2}+d \tilde{y}^{2}\right)=-d \tau^{2}+\tau^{2} d \chi^{2}=-d X^{2}+d Y^{2} \\
\left(\square-c_{\Lambda}\right) \Psi & =e^{-2(n+m) x}\left[-\frac{\partial^{2}}{\partial x^{2}}+\frac{\partial^{2}}{\partial \tilde{y}^{2}}-c_{\Lambda} e^{2(n+m) x}\right] \Psi=0
\end{aligned}
$$

where $\tau=\frac{1}{n+m} e^{(n+m) x}=\frac{z}{\sqrt{c_{\Lambda}}}, \chi=(n+m) \tilde{y}, X=\tau \cosh \chi, Y=\tau \sinh \chi$, and $\square$ is a d'Alembertian in the Milne metric . ${ }^{[26,27]}$

Following Ref. [27], let us define two vacua:

$$
\mid 0, \text { conf }\rangle=\mid 0, \text { in }\rangle, \quad \mid 0, \text { adia }\rangle=\mid 0, \text { out }\rangle \cdot{ }^{[29]}
$$

According to Ref. [27], the first vacuum $\mid 0$, con $f\rangle$ becomes the conformal vacuum in the limit $\Lambda \rightarrow 0$, the second vacuum $\mid 0$, adia $\rangle$ is the adiabatic vacuum and a comoving observer who has proper time $\tau \propto X$ will see no created universe in this vacuum. So, if we choose $\mid 0$, adia $\rangle$ as the initial state, no universe will be created and compactification will not be able to be explained even in the case that $\mathrm{n}=\mathrm{m}=3$ and $M^{3}=R^{3}, k_{3}=0$. It seems that further investigation will be needed on which vacuum should be preferred. 


\section{Summary}

We have studied on the third quantization of a Kaluza-Klein toy model, in which time $(x)$ is defined by the scale factor of universe, and the space coordinate $(y)$ is defined by the ratio of the scales of the ordinary space and the internal space. We calculated the number density of the universes created from nothing and found that there is a possibility to explain the compactification using the third quantization, when both the external and internal spaces are three-dimensional flat space $R^{3}$. However, our discussion is model dependent, and further study will be necessary.

\section{Acknowledgments}

The author would like to thank Prof. C. Isham, Prof. T.W.B. Kibble, Dr. J.J. Halliwell, Prof. A. Hosoya and Prof. T. Kitazoe for valuable discussions and encouragement. He would also like to thank Imperial College for hospitality where a part of this work was done. This work was supported in part by Japanese Ministry of Education, Science, Sports and Culture. 


\section{References}

[1] C.J. Isham, in Integrable Systems, Quantum Groups, and Quantum Field Theories, eds. L.A. Ibort and M.A. Rodriguez (Kluwer, London, 1993); K.V. Kuchař, in Proceedings of the 4th Canadian Conference on General Relativity and Relativistic Astrophysics, eds. G. Kunstatter, D.E. Vincent and J.G. Williams (World Scientific, Singapore, 1992).

[2] See also, e.g., H. Kodama, Prog. Theor. Phys. 94, 475 (1995); ibid. 94, 937 (1995); P. Hájiček, Nucl. Phys. B, Proc. Suppl. 57, 115 (1997); J.B. Hartle and D. Marolf, Phys. Rev. D 56, 6247 (1997); S. Kauffman and L. Smolin, "A Possible Solution to the Problem of Time in Quantum Cosmology", gr-qc/9703026; R. Brout and R. Parentani, "Time in Cosmology", gr-qc/9705072.

[3] J.J. Halliwell, in Quantum Cosmology and Baby Universes, eds. S. Coleman, J.B. Hartle, T. Piran and S. Weinberg (World Scientific, Singapore, 1991).

[4] T. Banks, Nucl. Phys. B 309, 493 (1988).

[5] S. Giddings and A. Strominger, Nucl. Phys. B 321, 481 (1989).

[6] M. McGuigan, Phys. Rev. D 38, 3031 (1988); ibid. 39, 2229 (1989).

[7] A. Hosoya and M. Morikawa, Phys. Rev. D 39, 1123 (1989).

[8] V.A. Rubakov, Phys. Lett. B 214, 503 (1988).

[9] W. Fischler, I. Klebanov, J. Polchinski and L. Susskind, Nucl. Phys. B 327, 157 (1989).

[10] Y. Xiang and L. Liu, Chinese Phys. Lett. 8, 52 (1991).

[11] H. Pohle, Phys. Lett. B 261, 257 (1991).

[12] S. Abe, Phys. Rev. D 47, 718 (1993). 
[13] T. Horiguchi, Phys. Rev. D 48, 5764 (1993).

[14] M.A. Castagnino, A. Gangui, F.D. Mazzitelli and I.I. Tkachev, Class. Quantum Grav. 10, 2495 (1993).

[15] A. Vilenkin, Phys. Rev. D 50, 2581 (1994).

[16] T. Appelquist, A. Chodos and P.G.O. Freund, Modern Kaluza-Klein Theories (Addison-Wesley, Reading, 1987).

[17] Z.C. Wu, Phys. Lett. B 146, 307 (1984); X.M. Hu and Z.C. Wu, ibid. 149, 87 (1984); Z.C. Wu, Phys. Rev. D 31, 3079 (1985).

[18] Y. Okada and M. Yoshimura, Phys. Rev. D 33, 2164 (1986).

[19] J.J. Halliwell, Nucl. Phys. B 266, 228 (1986); ibid. 286, 729 (1987).

[20] U. Carow-Watamura, T. Inami and S. Watamura, Class. Quantum Grav. 4, 23 (1987).

[21] Y. Zhong and X. Li, Phys. Rev. D 42, 712 (1990).

[22] F. Mellor, Nucl. Phys. B 353, 291 (1991).

[23] A. Zhuk, Class. Quantum Grav. 9, 2029 (1992).

[24] E.I. Guendelman and A.B. Kaganovich, Phys. Lett. B 301, 15 (1993).

[25] A.I. Zhuk, Physics of Atomic Nuclei 58, 1993 (1995).

[26] A.I. Zhuk, Gravitation \&3 Cosmology 2, 17 (1996).

[27] N.D. Birrell and P.C.W. Davies, Quantum Fields in Curved Space (Cambridge Univ. Press, Cambridge, 1982) .

[28] Following papers also examined another possibility to explain the compactification statistically: R.C. Myers, Nucl. Phys. B 323, 225 (1989); Y. Ohkuwa and W. Ogura, Prog. Theor. Phys. 84, 164 (1990). 
[29] See also, K. Yamamoto, T. Tanaka and M. Sasaki, Phys. Rev. D 51, 2968 (1995). 